\title{
Network Game Approach on Negotiation Process For the Case of the Caspian Oil Allocation
}

\author{
Maiko Sakamoto \\ Department of Civil Engineering \\ Nagasaki University \\ Nagasaki, Japan \\ sakamoto@civil.nagasaki-u. ac.jp
}

\author{
Kazuhiro Sasaki \\ Department of Civil and Environmental Engineering \\ Tohoku University \\ Sendai, Japan \\ k-sasaki@cneas.tohoku.ac.jp
}

\begin{abstract}
A methodology is shown to analyze phases of player's coalition formation. It can be applied to inter-regional cooperation analysis which is a relevant problem in Japan. Network formation game is used to model the problem with some extensions on a concept of coalition and coalition formative condition. A coalition among more than 3 players is introduced, which is not considered in standard network formation game. A coalition formative condition is also introduced taking into account specific characteristics about infrastructure construction. The methodology is applied to oil allocation problem among Caspian coastal countries, and how the methodology works in the real problem is examined. As a result, it is revealed that considering the coalition among more than 3 players is not sure to produce more social surplus than considering only the coalition between 2 players.
\end{abstract}

Keywords - Network game, the Caspian Negotiaon, Coalition, Allocation, Network Infrastructure Planning

\section{INTRODUCTION}

If there is an ongoing negotiation, what can scientists do for stakeholders to support their decision achieving a better solution for each of them? GMCR (Graph Model for Conflict Resolution [1]) is one of tools to support players' making decisions, which is based on non-cooperative game theory. In one hand, negotiation can be thought as a broader concept than conflict management because negotiation does not matter whether there exists a conflict between stakeholders or not. On the other hand, negotiation can be said as a narrower concept than conflict management because negotiation presumes a contact between stakeholders while non-cooperative game theory can deal with pre-negotiation situation where stakeholders are going to make a decision on whether they will communicate with other stakeholders or not.

Theoretical approach like game theory is sure to be a powerful tool for stakeholders in negotiation and/or conflict to help them understand clearly their situation. However, if it provides more than single equilibrium, in other words, if there exist several equilibria which lie in Pareto optimum, it does not give stakeholders any instructions for managing the conflict. That is, showing a structure of a conflict does not necessarily mean shrinking complexity, but it just does showing how complex the system is. Since a role of a system is to shrink complexity referring to Luhmann ([2]), fields of negotiation and/or conflict management should be elaborated more scientifically and provide theoretical framework to achieve shrinking complexity for stakeholders facing with negotiation and/or a conflict. In other words, fields of negotiation and/or conflict management are now required to be a system for supporting stakeholders' decision-making.

In this paper, to contribute to this purpose, network game is transformed to apply a real case. Although network game incorporates both concepts of non-cooperative game theory and cooperative game theory, this paper focuses on non-cooperative game aspect. We show how our set of models works to shrink complexity of the real ongoing negotiation in the Caspian problem.

Participatory decision-making should be taken in negotiation and/or conflict management process where other individuals except players, neither of facilitator or scientists involved as advisors, cannot induce players to a certain goal if there are more than single equilibrium which are Pareto relationship, and this is the usual case. In this case, roles of scientists will be to reveal issues and strategies which are strongly dominated by other issues and strategies. This will be a help for stakeholders because it shrinks complexity, and this is what this paper aims at.

\section{Network GAME AND ITS EXTENSION}

\section{A. Network Game[3]}

Network game aims to give strategic foundations to network formation. Coalitions are groups of players whose members decide to cooperate, possibly by establishing binding agreements. Networks are described by bilateral links between decision makers (nodes or players). In a cooperative game, since coalitions are sets of players, how players are connected does not matter. Therefore, network game can express more variety of coalitions. The precise structure of the links connecting a coalition may influence the payoffs to its members.

$N=\{1,2, \ldots, n\}$ is a set of players of individuals connected in some network relationship. These players will be the nodes or vertices in a graph that will describe the network relationships. Each player has discretion in forming his or her links in the network relationship. These may be people deciding on whom they wish to contract with. Two players are 
either related to each other or not, but it cannot be that one is related to the second without the second being related to the first. Joint consent is needed to establish and maintain the link.

A network $g$ is a list of which pairs of players are linked to each other. A network is then a list of unordered pairs of players $\{i, j\}$. For any pairs of players $i$ and $j,\{i, j\} \in g$ " indicates that $i$ and $j$ are linked under the network $g$. For simplicity, write $i j$ to represent the link $\{i, j\}$, and so $i j \in g$ " indicates that $i$ and $j$ are linked under the network $g$. For instance, if $N=\{1,2,3\}$, then $g=\{12,23\}$ is the network in which there is a link between players 1 and 3 .

Let be $g^{N}$ be the set of all subsets of $N$ of size 2, $G=$ $\left\{g \subset g^{N}\right\}$ denotes the set of all possible networks of graphs on $N$. The network $g^{N}$ is referred to as the "complete" network.

A shorthand notation for the network obtained by adding link $i j$ to an existing network $g$ is $g+i j$, and for the network obtained by deleting link $i j$ from an existing network $g$ is $g-i j$.

A network game is a pair $(N, v)$, where $N$ is the set of players and $v$ is a value function on networks among those players.

Jackson and Wolinsky ([4]) defines pairwise stable to model network formation by dispensing with the specifics of a non-cooperative game and simply model a notion of what a stable network is directly.

Pairwise stability: A network $g$ is pairwise stable with respect to allocation rule $\pi$ and value function $v$ if

(i) for all $i j \in g$,

$$
\pi_{i}(g, v) \geq \pi_{i}(g-i j, v) \text { and } \pi_{j}(g, v) \geq \pi_{j}(g-i j, v) \text {, and }
$$

(ii) for all $i j \notin g$,

$$
\text { if } \pi_{i}(g, v)<\pi_{i}(g+i j, v) \text {, then } \pi_{j}(g, v)>\pi_{j}(g+i j, v) \text {. }
$$

The first part of the definition of pairwise stability requires that no player wish to delete a link that he or she is involved in. Implicitly, any player has the discretion to unilaterally terminate relationships in which he or she is involved. The second part of the definition requires that if some link is not in the network and one of the involved players would benefit from adding it, then it must be that the other player would suffer from the addition of the link. Here, it is implicit that the consent of both players is needed for adding a link.

Although pairwise stability is natural and quite easy to work with, it is a so week notion that it can consider deviations by at most a pair of players at a time. Pairwise stability might be thought of as a necessary but not sufficient requirement for a network to stable over time.

Alternative to pair wise stability that allow for larger collations than just pairs of players to deviate were first considered by Dutta and Mutuswami([5]). The following definition is in that spirit and is due to Jackson and van den Nouweland([6]). if

A network is obtained from $g^{\prime} \in G$ via $g \in G$ deviation by $S$ (i) $i j \in g^{\prime}$ and $i j \notin g$ implies $i j \subset S$, and

(ii) $i j \in g$ and $i j \notin g^{\prime}$ implies $i j \cap S \neq \varnothing$.

This definition identifies changes in a network that can be made by a coalition $S$ without the consent of any players outside of $S$. Part (i) requires that any new links that are added can only be between players in $S$. This requirement arises because the consent of both players is needed to add a link. Part (ii) requires that at least one player of any deleted link be in $S$. This is because either player in a link can unilaterally sever the relationship.

Strong stability: A network is strongly stable with respect to allocation rule $\pi$ and value function $v$ if for any $S \subset N, g^{\prime}$ that is obtained from $g$ via deviations by $S$, and $i \in S$ such that

$$
\pi_{i}\left(g^{\prime}, v\right)>\pi_{i}(g, v),
$$

there exists $j \in S$ such that

$$
\pi_{j}\left(g^{\prime}, v\right)<\pi_{j}(g, v) .
$$

Strong stability provides a powerful refinement of pairwise stability. It mainly makes sense in smaller network situations in which players have substantially information about the overall structure and potential payoffs and can coordinate their actions. As strong stability is a very demanding concept, what appears to be an improving deviation might not be taken if one started to forecast how the other players might react. Nevertheless, when strongly stable networks exist, they have very nice properties.

\section{B. Extention of Network Game and Strategies for Coallition}

In network game, multilateral coalition is assumed as a pile of bilateral coalition while it is assumed just as a set of players in cooperative game theory. These are thought to be complementary properties of coalition. Therefore, in this paper, the concept of coalition in cooperative game theory is introduced to network game framework as an extension, which we will call partial grand coalition in this paper.

Players in partial grand coalition are assumed to maximize their aggregate benefit, and network is decided by players in the partial grand coalition to fulfill their maximization. Since, in this paper, coalition is regarding whether a network infrastructure is constructed or not between particular pairs of players, the partial grand coalition will decided a infrastructure network where there does not necessarily exist a infrastructure between certain pairs of players in the coalition.

Now, terms seem to be so complicated that a summary of the terms is shown in Table 1. Fig.2 will be also a help to understand the relationship. In a bilateral coalition case, a link has two meanings at the same time. That is, if there is a link between a certain pair of players, they have made a coalition between them, and eventually they have constructed an infrastructure between them. In partial grand coalition case, a link has an only meaning that there exists an infrastructure as a result of aggregate decision by the players in the partial grand coalition. As for a description about a coalition, coloring node is used for the case of partial grand coalition, which is shown in Fig.2. Example 1 shows that player 1, 2, 3 have made a partial 
TABLE I. REALTIONSHIP OF TERMS

\begin{tabular}{|c|c|c|}
\hline $\begin{array}{l}\text { Graph } \\
\text { Theory }\end{array}$ & \multicolumn{2}{|c|}{ Interpretation of Components } \\
\hline $\begin{array}{l}\text { Description } \\
\text { in graph }\end{array}$ & Non-physical Relationship & Physical Relationship \\
\hline link & $\begin{array}{c}\text { Coalition in Birateral } \\
\text { Negotiation }\end{array}$ & Single Infrastructure \\
\hline coloring node & $\begin{array}{c}\text { Coalition in Multirateral } \\
\text { Negotiation }\end{array}$ & - \\
\hline graph & Coalition Structure & $\begin{array}{l}\text { Network of } \\
\text { Infrastructures }\end{array}$ \\
\hline
\end{tabular}

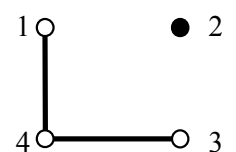

Ex.1

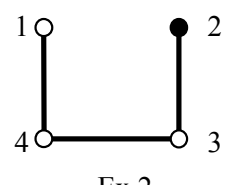

Figure 1. Visual Explation of Terms

grand coalition, and as a result of aggregate decision-making, links have been constructed between player 1 and 4, 3 and 4 . Player 2 is not in any coalitions. Example 2 shows that player 1, 2, 3 have made a partial grand coalition, and player 2 and 3 have made a bilateral coalition. As a result of aggregate decision-making by player $1,3,4$, infrastructures are constructed between player 1 and 4, 3 and 4. As for the bilateral coalition between 2 and 3, an infrastructure has been constructed between player 2 and 3. Therefore, a link has double meanings in the case of bilateral coalition. Graphs of example 1 and 2 give visual information about coalition structure and infrastructure network formations at the same time.

\section{Coalition Foramative Condition}

As explained in the section A of this chapter, pairwise stability or strong stability can be a coalition formative condition in network game. Based on real cases of infrastructure construction, we can establish another coalition formative condition. In the following, we assume that allocation rule $\pi$ does not account initial cost but only running cost.

Coalition formative condition for bilateral coalition: A network $g+i j$ is formative with respect to allocation rule $\pi$ and value function $v$ if for all $i j \in g, \pi_{i}(g+i j, v)>\pi_{i}(g, v)$ and $\pi_{j}(g+i j, v)>\pi_{j}(g, v)$.

Coalition formative condition for multilateral coalition: A network $g$ ' where links and nodes are added to $g$ is formative with respect to allocation rule $\pi$ and value function $v$ if for all $i \in g^{\prime}, \pi_{i}\left(g^{\prime}, v\right)>\pi_{i}(g, v)$.

These conditions consider irreversibility and initial cost of construction. Players cannot remove the infrastructure which they have constructed so that conditions do not mention anything about reduced graph $g-i j$. As for the initial cost of constructing a infrastructure, it is usually so huge amount of money that both of conditions do not contain equal signs because payoff should be lager in the graph $g+i j$ or $g$ ' than in graph $g$ on the assumption of allocation rule $\pi$ which does not consider initial cost.

\section{THE CASPIAN PROBLEM}

The framework of analysis discussed in the previous chapter is applied to on-going negotiation problem among littoral countries of the Caspian. Before showing details of model and analysis results, background of the problem is briefly explained.

The Caspian is known as the largest lake in the world. Surface area is $371,000 \mathrm{~km}^{2}$, volume is $78,200 \mathrm{~km}^{3}$, maximum depth is about $1,025 \mathrm{~m}$, and salinity is approximately $1.2 \%$. Seeing the salinity ratio, it can be thought as sea. However, there is no particular international law which can be applied to the Caspian. Technically, the definition of lake is that the water body is not connected to the open seas, and on the other hand, the definition of sea is that the water body is connected to the open seas. The Caspian is not naturally connected to any open seas, but it is artificially connected to Black Sea via Volga-Don canal. Therefore, international law of lake or sea cannot strictly apply to the Caspian. Before USSR was disintegrated, there were only two countries around the Caspian, namely USSR and Iran. They agreed on treating the Caspian as lake. In other words, the Caspian used to be divided into two parts between USSR and Iran based on the ordinal way of regulating lakes.

However, after USSR was disintegrated, the situation changed. The littoral countries are now five; Russia, Iran, Azerbaijan, Turkmenistan, and Kazakhstan. Depending on the regulation rule, the stakes of each country are so different that the littoral countries are claiming their own interests, and they have recently just started negotiating with each other in the dialog initiated by International Institute for Applied Systems Analysis in Austria.

The Caspian problem is complicated because there are lots of issues to be agreed, which are related each other. Is comprehensive agreement better than piling up single-issue agreements? To answer the question, we need to understand the system of the Caspian problem at first.

Agreement on oil allocation can be thought as a critical issue which lies on the basis of the comprehensive problem. Therefore, the attitude of players toward solving energy negotiation may decide the attitude toward solving other issues. However, energy issue has not been discussed well among five countries although some bilateral agreements have been concluded, and this can be thought as a reason why the Caspian negotiation seems to be prolonged.

Now, we are going to model the oil conflict by the extended network game, and a certain portion of complexity will be reduced. This will be somehow a help of solving whole issues remaining.

Players are set as Russia, Azerbaijan, Kazakhstan, and EU because Caspian oil is basically exported to EU so that the related stakeholders in oil trade involving EU are considered. In the context of oil allocation, the infrastructure means pipeline. Therefore, our main focuses in the following are on how to construct pipelines, how to allocate oil through the constructed pipelines and how the coalition structure and 
pipeline network will evolve. We will consider all the possibilities of pipelines which are represented by all the combinations of two players unless the two cannot be connected directly because of certain topographical reasons.

\section{OIL ALlOCATION MOdeL}

In this chapter, models for calculating payoffs are established. As notations, $g$ is used as coalition structure and pipeline network for cases of bilateral approach while $g s$ is used for cases of multilateral approach.

\section{A. Allocation by birateral collaiiton between 2 players in} birateral coallition

It is assumed that there exist pipelines between player $i(i \in N)$ and player $j(j \notin i)$, player $i$ and player $k$ $(k \notin i, k \notin j)$. Player $k$ can produce oil, and it exports oil to player $i$. Player $i$ can export oil to player $j$ if it imports oil from player $k$ and/or it produces oil by itself. Player $i$ decides the amounts of its production, domestic supply and export to player $j$ by maximizing its own profit. The situation is depicted in Fig.2.

Player $i$ 's oil is defined as sum of 1) domestic produced oil and 2) imported oil from other players except player $j$ in the situation of Fig.2. In the case of 1), player $i$ can produce oil by $X_{i}$ with adjusting the amount to satisfy domestic demand at most $X_{i \max }$ during a certain period. If player cannot produce oil then $X_{i}=0$. In the case of 2), sum of imported oil to player $i$ is defined as in equation (1).

$$
Y_{i}=\sum_{k \in N_{i}^{\prime}} Y_{k i}
$$

Note that $N_{i}{ }^{\prime}=\{k \mid k \in N, k \neq i, k \neq j\}$.

Player $i$ 's oil can be represented by $X_{i}+Y_{i}$. The oil exported to player $j$ is defined by $q_{i j}$, and the oil supplied to its own country is defined by $q_{i}$. Domestic oil price and demand of player $i$ are represented by $p_{i}, z_{i}$ respectively while domestic oil price and demand of player $j$ are represented by $p_{j}, z_{j}$. Now we can describe the relationship of domestic oil price and demand as equations (2) and (3) which are inverse demand functions.

$$
\begin{gathered}
p_{i}=D_{i}\left(z_{i}\right) \\
p_{j}=D_{j}\left(z_{j}\right)
\end{gathered}
$$

Considering the situation of Fig.2, the general description of inverse demand functions are redefined as equations (4) and (5).

$$
\begin{gathered}
p_{i}=D_{i}\left(q_{i}\right) \\
p_{i j}=D_{j}\left(q_{i j}\right)
\end{gathered}
$$

Transportation cost per unit from player $i$ to player $j$ is defined as $c_{i j}$, and production cost per unit of player $i$ is defined as $c_{i}$.

With the model settings, the problem to solve can be defined as equation (6) where player $i$ decides the amount of

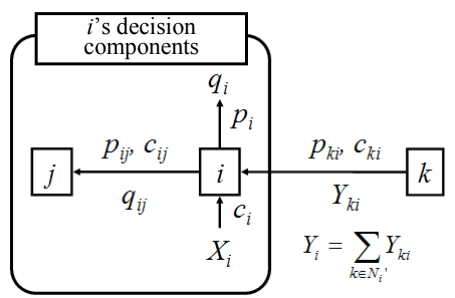

Figure 2. Model of Birateral Collaiiton between 2 Players

domestic production $X_{i}$, domestic supply $q_{i}$ and exported oil production $X_{i}$, domestic supply $q_{i}$ and exported oil to player $j q_{i j}$ to maximize its own profit $\pi_{i}$.

$$
\begin{aligned}
& \pi_{i}=\max _{q_{i j}, q_{i}, X_{i}}\left\{\sum_{j \in N_{i}} p_{i j} q_{i j}+p_{i} q_{i}\right. \\
& \left.\quad-\sum_{j \in N_{i}} c_{i j} q_{i j}-\sum_{k \in N_{i}^{\prime}} p_{k i} Y_{k i}-c_{i} X_{i}\right\} \\
& \text { s.t. } \sum_{j \in N_{i}} q_{i j}+q_{i}=X_{i}+Y_{i}, \\
& \quad q_{i j} \geq 0, q_{i} \geq 0,0 \leq X_{i} \leq X_{i \max }
\end{aligned}
$$

Note that $N_{i}=\{j \mid j \in N, j \neq i, j \neq k\}$.

\section{B. Allocation among more than 3 players in partial grand coallition}

The base model of deciding oil allocation among more than 3 players is same as the model for 2 players in the previous section. However, in the model for 3 players, a certain player can export oil to another player in the coalition who is not directly connected but indirectly connected via a certain player in the partial grand collation. The objective of partial grand coalition is to maximize sum of profit of all the players in the coalition so that any pair of players can trade with each other with the pipelines which are shared by all the players in the coalition. Note that price of oil does not change if the oil only passes through the players who are not traded with.

The maximization problem can be described as equation (7).

$$
g s=\left\{g^{\prime} \mid \max _{g^{\prime} \in G R^{N^{\prime \prime}}}\left(\max \sum_{i \in N^{\prime \prime}} \pi_{i}\left(g^{\prime}\right)\right)\right\}
$$

where sum of profit of all the players in the partial grand coalition is maximized by constructing pipelines. In other words, the partial grand coalition defines a certain network $g s$ out of the remaining of $G R^{N^{\prime \prime}}$ which is the set of partial graphs of perfect graph $g^{N \prime}$ where $N^{\prime \prime}$ is the set of players in the partial grand coalition. 


\section{ANALLySis OF CALLITION Fromation Process}

\section{A. Data and Details of Model Settings}

To analyze the Caspian energy negotiation problem with the models discussed, parameters of inverse demand function, transportation cost, production cost and the maximum amount of produced oil are needed to be set referring to data.

At first, the inverse demand function $D_{i}$ is assumed as linear function which defines the relationship of domestic demand $z_{i}$ barrel/day and price $p_{i} \$ /$ barrel. It can be described as in equation (8).

$$
p_{i}=A_{i} z_{i}+B_{i} \quad\left(A_{i}, B_{i}: \text { constants }\right)
$$

Price elasticity $\mathcal{\varepsilon}$ for the inverse demand function is defined as equation (9).

$$
\varepsilon=\frac{1}{A_{i}} \cdot \frac{p_{i}}{z_{i}}
$$

Once a pair of a certain price and demand and price elasticity are observed, the inverse function is obtained by solving the equations (8) and (9).

As for the price elasticity $\mathcal{E}$, the estimated average value of price elasticity in long term to demand for gasoline in United States is used, namely -0.38 ([7]). The price is set as the average oil price in 2006 referring to the report of oil company BP ([8]). The demand is set using the same report with consideration on players' energy situations. The demand of Azerbaijan and Kazakhstan are set based on the report. Russian demand is set as weighted demand by population. That is, the imported oil to Russia from the Caspian is thought to be distributed only in south region where its population ration to whole population in Russia is 0.16 ([9]). Then, 0.16 is multiplied with total demand of oil in Russian, and this is used for Russian demand in this region. As for EU, the demand is set by multiplying total consumption and 0.27 which is import ration from Russia ([10]).

By solving the equations (8) and (9) with the settings above, the estimated constants $A_{i}$ and $B_{i}$ and corresponding values of price and demand are obtained as Table 2 .

As for the transportation cost of oil, $4 \$ /$ barrel is used for any links based on the transportation cost of CPC pipeline reported by IDCJ ([11]). This is because the assumed players, namely Russia, EU, Azerbaijan, Kazakhstan, are situated closely so that there does not exist much difference in topography, geology and climate which may give the difference to the transportation cost.

The same logic can be applied to the production cost of oil. Therefore, $7 \$$ barrel is used as all the players' production cost, which is the production cost of Kazakhstan reported by Cambridge Economic Research Association.

As for the maximum amount of oil production in a certain period, the produced amount in 2006 is used, which is reported
TABLE II. ESTIMATED VALUES OF INVERSE DEMAND FUNCTION

\begin{tabular}{|l|r|r|}
\hline & \multicolumn{1}{|c|}{ Russia } & \multicolumn{1}{|c|}{ EU } \\
\hline$p$ (\$/barrel) & 65.14 & 65.14 \\
\hline$z$ (barre1/day) & 437616.29 & 4318403.03 \\
\hline$A$ & -0.000392 & -0.000040 \\
\hline$B$ & 236.58 & 236.58 \\
\hline & Kazakhstan & Azerbaijan \\
\hline$p$ (\$/barrel) & 65.14 & 65.14 \\
\hline$z$ (barre1/day) & 220743.01 & 96467.48 \\
\hline$A$ & -0.000777 & -0.001777 \\
\hline$B$ & 236.58 & 236.58 \\
\hline
\end{tabular}

by oil company BP in 2006 . Therefore, $1.43 \times 10^{6}$ barrel/day is used for Kazakhstan and $0.65 \times 10^{6}$ barrel/day is used for Azerbaijan. Russia and EU do not have their own fields so that the maximum amounts of oil production for them are not set.

\section{B. The case of birateral approach}

Now, coalition formation process on the assumption that players consider only bilateral approach is analyzed.

To evaluate coalition structures, sum of social surplus $S C(g)$ is used, which is defined in equation (10)

$$
S C(g)=\square_{i \in N(g)} S C_{i}(g)=\square_{i \in N(g)}\left(\pi_{i}(g)+C S_{i}(g)\right)
$$

$S C_{i}(g)$ : Social surplus of player $i$ in the coalition structure $g$

$\pi_{i}(g)$ : Profit of player $i$ in the coalition $g$

$C S_{i}(g)$ : Domestic consumer surplus of player $i$ in the coalition structure $g$

$N(g)$ : The set of players in the coalition structure $g$

The coalition formation process can be obtained as Fig. 3 and some examples of coalition structure are depicted in Fig.4. In Fig.3, $\mathrm{x}$-axis shows the number of constructed pipelines, and $y$-axis shows sum of social surplus calculated by equation (10).

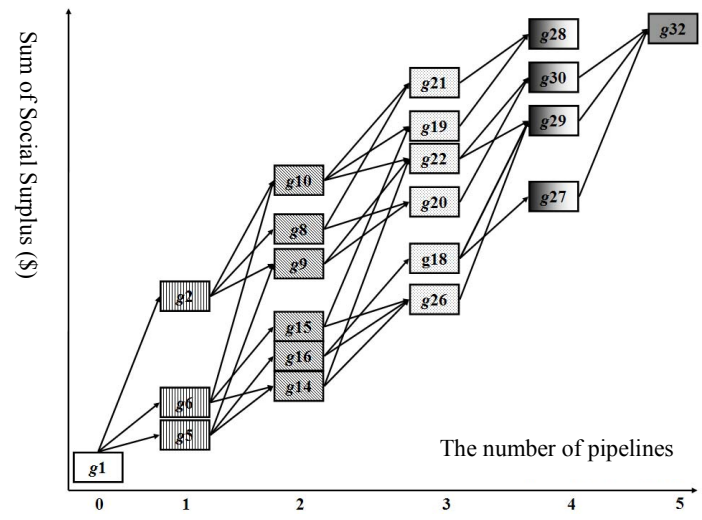

Figure 3. Coaltion Formation Process in the case of Birateral Approach 


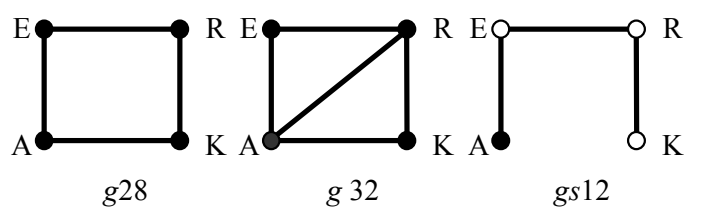

Figure 4. Examples of Coalition Structures

In Fig.4, R, K, A, and E represent Russia, Kazakhstan, Azerbaijan, and EU respectively. As seen in Fig. 4, in the case that players negotiate only bilaterally, $g 28$ and $g 32$ are obtained as stable coalition structures which do not have any transferable coalition structures. Comparing these two coalition structures, $g 32$ requires one more additional pipeline than $g 28$ to achieve the same level of social surplus. Installing cost of pipelines is huge and it will obviously pollute environments so that $g 28$ is desirable from a view point of social norm. However, it can be observed in Fig. 3, there exists path dependency, and as the earliest example, if players decide to choose the situation of $g 9$, they can never reach $g 32$ afterwards.

\section{The case of multirateral approach}

Finally, the coalition formation process in the case of multilateral approach is analyzed. The assumption is that that players consider both of bilateral coalition and partial grand coalition as their strategies. Corresponding to this assumption, $S C(g)$ in the equation (10) is redefined $S C(g)$ or $S C(g s)$ where $g$ represents the bilateral approach, and $g s$ represents the multilateral approach which considers both of bilateral coalition and grand partial coalition. The analysis result is shown in Fig.5

Seeing Fig.5, there exist some multilateral approach paths which provide more profit than bilateral approach paths do. Furthermore, gs 12 and $g s 21$ which provide the highest profit can be reached with quite a few steps. In our model, the negotiation cost is not considered, but once seeing the result of Fig.5, gs 12 and gs 21 have a good property regarding the negation cost as well as the high level of social surplus.

\section{CONCLUSION}

In this paper, the methodology to analyze the coalition formation process regarding construction of network infrastructure is proposed. The framework of network game is applied with some extensions on the concept of coalition and coalition formative conditions.

As a result, the coalition formation processes for the Caspian oil negotiation are obtained for the two cases; one is the case where players consider only bilateral approach, and another is the case where players consider both of bilateral coalition and partial grand coalition which we call multilateral approach. Usually, it is thought that aggregate decision-making is better than piles of bilateral approach but it is not necessarily true as we can see in Fig.5.

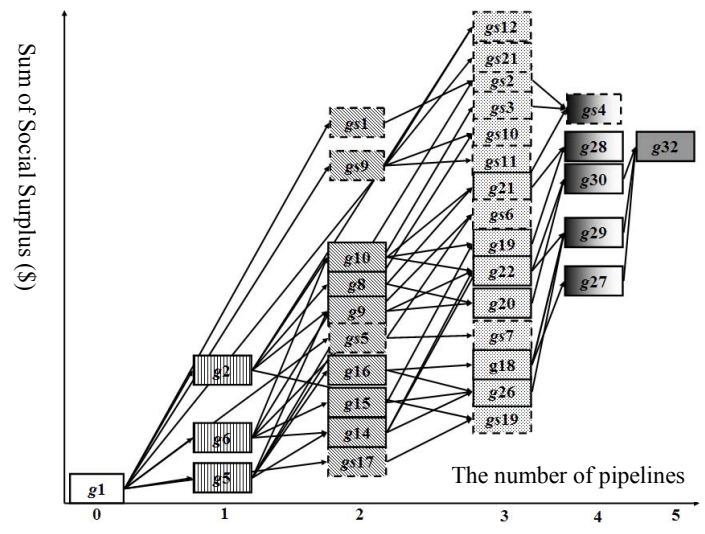

Figure 5. Coaltion Formation Process in the case of Birateral Approach

The information is not usually symmetry among players. It is needless to say that the information is also asymmetry between players and scientist or coordinators who are third parities. Therefore, an important role of scientists and coordinators is not to point out the resolution but to shrink complexity for players to make a decision by themselves. In addition to this, it is a ideal situation if the instruction provided by scientist and coordinators motivates players to share more information among them. The models shown in this paper may contribute somehow to this purpose.

The cost allocation model and considering equity in allocation are remaining tasks to be dealt with in near future.

\section{REFERENCES}

[1] M. O. Jackson, "A Survey of Models of Network Formation: Stability and Efficiency," Group Formation in Economics (edited by G. Demange, M. Wooders), pp.11-57, 2005

[2] N. Luhmann, "Soziale Systeme Grundrib einer allgemeinen Theorie," Suhrkamp, 1984

[3] L. Fang, K.W. Hipel, and D.M. Kilgour, "Interactive Decision Making: The Graph Model for Conflict Resolution", Wiley, New York, 1993.

[4] M.O. Jackson and A. Wolinsky, "A Strategic Model of Social and Economic Networks," Journal of Economic Theory, Vol. 71, pp. 44-74, 1996.

[5] B. Dutta and S. Mutuswami, "Stable Networks," Journal of Economic Theory, Vol.76, pp. 322-344, 1997.

[6] M.O. Jackson and A. van den Nouweland, " Storongly Stable Networks," forthecoming: Games and Economic Behavior, http://www.hss.caltech.edu/ jacksonm/coopnet.pdf, 2000.

[7] BP, Statistical Review of Worl, June 2007.

[8] Russian Federal National Statistics Committee, http://www.gks.ru/, (2007.12.4).

[9] Fujitsu Research Insittute, http://jp.fujitsu.com/group/fri/ report/ conomic-review/200301/page16.html, (2008.1.10).

[10] IDCJ, http://www.idcj.or.jp/1DS/11 ee jousei021106.htm, (2008.1.10).

[11] Cambridge Economic Research Association, 2001. 